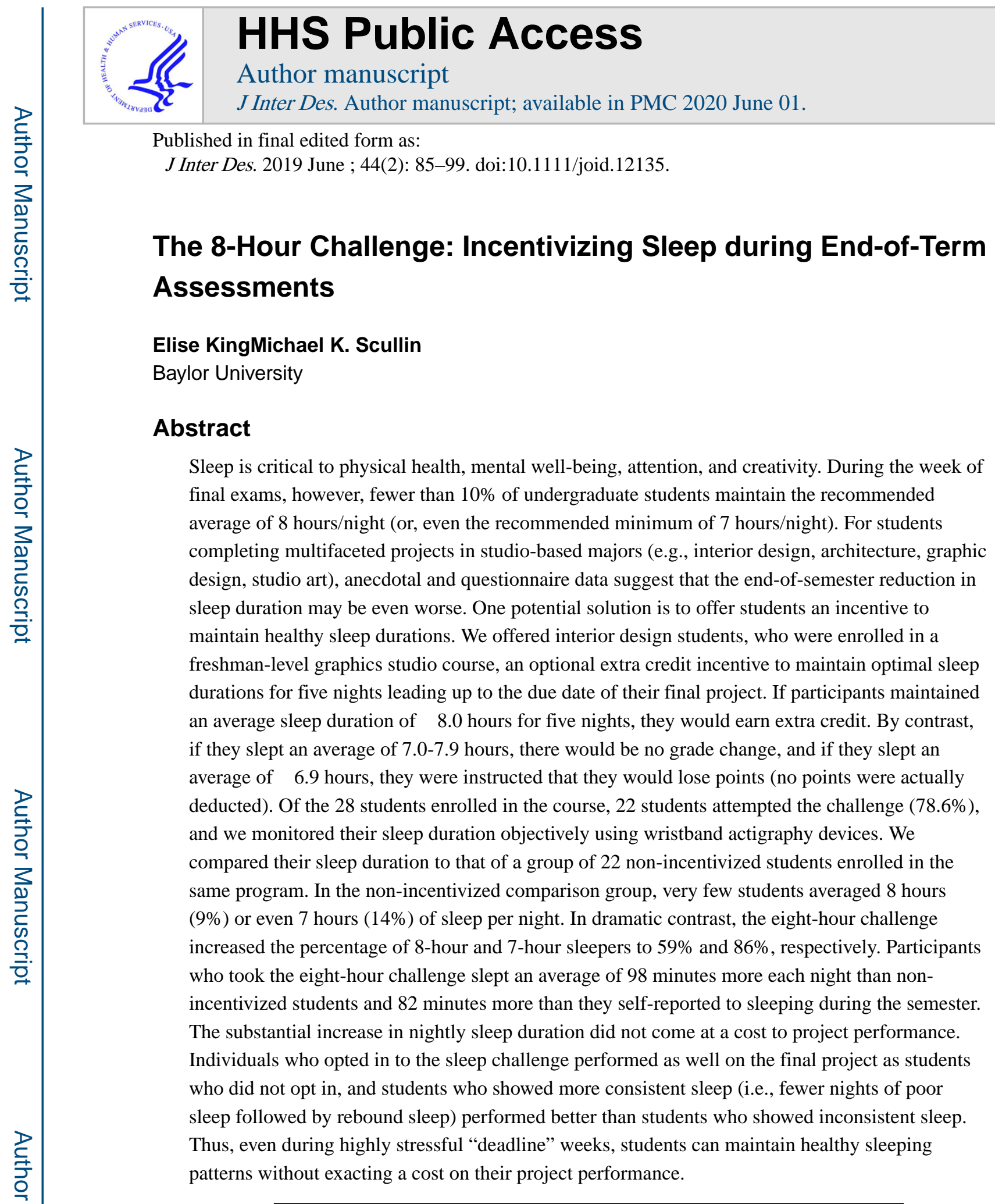

\title{
Introduction
}

Undergraduate students are notorious for their poor sleep habits (Lund, Reider, Whiting, \& Prichard, 2010). Within studio-based courses (e.g., interior design, studio art, and

Correspondence: Michael K. Scullin, Ph.D., Baylor University, Department of Psychology \& Neuroscience, One Bear Place \#97334, Waco, TX 76798, Michael_Scullin@Baylor.edu or Elise_King@baylor.edu. 
architecture), data suggest that students may cycle between nights of restricted (short) sleep and recovery (long) sleep (King, Daunis, Tami, \& Scullin, 2017). Students often restrict sleep with the expectation that studying or working on projects late into the night will lead to a better grade. Some correlational studies show that students who restrict their sleep are more likely to have lower grade point averages (Phillips et al., 2017; Smarr, 2015; Trockel, Barnes, \& Egget, 2000; Wolfson \& Carskadon, 2003). One explanation for why spending more hours preparing for a project (but fewer hours sleeping) does not benefit overall performance is that short and irregular sleep patterns reduce work efficiency, spatial reasoning, and creativity (Budnick \& Barber, 2015; King et al., 2017). Thus, the supposed gains from spending extra hours awake to work on a project might be offset by the cognitive impairments that sleep loss induces.

Despite an abundance of data that confirm the importance of sleep to cognition and health, many students continue to engage in irregular and restricted sleep. Such poor sleep habits are often intensified during the week of final exams. As displayed in Figure 1, during final exams and final project reviews (or end-of-term presentations for studio-based courses), fewer than $10 \%$ of undergraduate students maintain optimal sleep durations (classically defined as eight hours per night, though some academic societies define optimal duration for adults as between seven and nine hours per night; Paruthi et al., 2016; Walker, 2017; Watson et al., 2017). In an effort to improve student sleep habits, universities and secondary schools have implemented sleep education programs. These programs increase students' knowledge about the importance of healthy sleep habits, but they have not proven to be effective in changing behavior (for review, see Blunden, Chapman, \& Rigney, 2012). As such, what can educators do to change student sleep habits, particularly during finals week? One possible solution is to provide students with an incentive to sleep better. Therefore, in the current study, we offered students an opportunity to earn extra credit for sleeping eight hours per night during finals week.

\section{Background Overview of Sleep}

Sleep is a reversible state characterized by closed eyes, immobility, and unresponsiveness to external stimuli (Carskadon \& Dement, 2017). Sleep, or sleep-like states, are universal across all, or nearly all, species (Hendricks, Sehgal, \& Pack, 2000; Lesku \& Rattenborg, 2014; Trojanowski \& Raizen, 2016), though species range from needing only a few hours of sleep per night (e.g., giraffes) to needing 16 or more hours (e.g., koalas). The question of how much sleep humans need has long been a source of popular debate (Scullin, 2017), in part, because sleep need changes from infancy to old age. For example, consensus guidelines state that adolescents need 8-10 hours of sleep/night whereas adults need 7-9 hours of sleep/ night (note that freshman college students, the population of interest in this study, are at the developmental intersection of adolescence and young adulthood; Paruthi et al., 2016; Watson et al., 2017). Many factors contribute to short sleep duration in college studentsnoisy roommates, electronic devices, stress, work, stimulants, and socializing — but regardless of reason, restricting sleep duration below the recommended guidelines increases risk for the common cold (Prather, Janicki-Deverts, Hall, \& Cohen, 2015), automobile 
accidents (Bryant \& Gomez, 2015), substance abuse (Roane \& Taylor, 2008), as well as poor dietary and physical activity habits (Nedeltcheva, Kessler, Imperial, \& Penev, 2009).

Furthermore, sleep deprivation is known to impair mood and emotional regulation (Haack \& Mullington, 2005). The ability to regulate emotions allows humans to enjoy positive experiences and limit the effects of negative emotions. Sleep deprivation, however, can hinder the ability to cope with emotional challenges and makes us more sensitive to painful emotional events (Vandekerckhove \& Cluydts, 2010). The prefrontal cortex, an area of the brain that regulates emotions, has been shown to be particularly susceptible to sleep loss (Scullin, 2017). In sleep deprived individuals, positron emission tomography (PET) scans show significant declines in activity within the prefrontal cortex as well as other areas associated with executive control and emotional processing, including the thalamus and parietal regions (Thomas et al., 2000). Furthermore, sleep deprivation is known to negatively impact sustained attention (Lowe, Safati, \& Hall, 2017), as well as learning and memory (Rasch \& Born, 2013). In other words, the effects of sleep loss are manifold, and cutting back on sleep impairs many basic processes that are foundational for academic performance.

\section{Sleep and Academic Performance}

Students generally perform best on exams when they space their studying across many days (e.g., Dempster \& Farris, 1990; Gillen-O’Neel, Huynh, \& Fuligni, 2013). Few students, however, engage in spaced learning; instead, most students tend to "cram" right before exams or other deadlines (Miyatsu, Nguyen, \& McDaniel, 2018). One consequence of procrastination and cramming is that students may restrict sleep with the intention of studying more. While that may seem to students like a good strategy, the costs of restricting sleep might outweigh the potential benefits of taking more time to study (Wolfson \& Carskadon, 1998). For example, Thacher (2008) reported that $60 \%$ of college students have "pulled an all-nighter" and that students who pulled more all-nighters had lower grade point averages (GPAs). Additionally, studies have found that though sleep-deprived students objectively performed worse and exerted more effort on cognitive tasks, they reported significantly higher levels of estimated performance than their non-deprived peers (Pilcher \& Walters, 1997). Therefore, college students may not be aware of the extent to which sleep restriction impairs their cognition (Van Dongen, Maislin, Mullington, \& Dinges, 2003), which may help to explain why students continue to engage in sleep-restricting habits.

\section{Sleep in Studio-Based Courses}

Students in studio-based courses often report forgoing sleep to meet project deadlines (Anthony, 1991; American Institute of Architecture Students Studio Culture Task Force, 2002). The studio model of instruction, which is prevalent in several academic disciplines, including interior design, art, architecture, and graphic design, utilizes project-based assessments (of varying lengths and durations). Students complete much of the work independently and are responsible for managing short- and long-term project deadlines in multiple courses. With some deadlines being weeks or months in the future, it is critical for students to work efficiently during all stages of a project. However, many universities allow 24-hour access to design studios, and as deadlines approach, students frequently limit their sleep to work on projects (Anthony, 1991; King et al., 2017). Survey data suggest that 
students in studio environments often show chronic sleep restriction consistent with clinical insomnia (Anthony, 1991; Bachman \& Bachman, 2006; Springett \& Lekarz, 1986).

Additionally, King et al. (2017) used actigraphy devices to measure objectively the sleep quantity and quality of 28 interior design undergraduates over a seven-day period and observed that most students do not sleep for eight hours (optimal), or even seven hours (minimal), every night. Furthermore, students who maintained short sleep durations or highly variable sleep patterns (e.g., restricting sleep and then rebounding on sleep) demonstrated pre-to-post week declines on measures of attention and creativity (King et al., 2017). Given the importance of attention and creativity to studio-based projects, King et al.'s results call into question the utility of restricting sleep in the days before a project deadline.

\section{Sleep and Final Reviews/Exams}

Poor sleep habits are often heightened when students are preparing for final exams or final project deadlines (Buboltz, Brown, \& Soper, 2001; Taylor \& Bramoweth, 2010; Wolfson \& Carskadon, 2003). Increased stress, excessive caffeine/stimulant use, and the 24/7 culture of many design studios lead to shorter sleep durations, "all-nighters," and sleep schedule variability (Ahrberg, Dresler, Niedermaier, Steiger, \& Genzel, 2012). In addition, studiobased courses often include the added pressure of oral critiques (or "crits"). If a student in a traditional lecture course performs poorly on a test, the negative result is known only to the student and the instructor. But, studio-based courses often involve a final project crit: a highstake oral presentation in which students present their project and receive feedback from faculty, peers, and guest professional reviewers. Crits have been the standard method of evaluation since the origin of design studios at the Beaux Arts (Anthony, 1991), but horror stories abound of grandstanding reviewers and clashing egos. Consider, for example, this description by Martin Pawley (former architecture critic of the Guardian) of his experience as an architecture student in London:

The student collapsed whilst his project was being energetically ridiculed by a visiting critic... At Oxford, girl students had sometimes burst into tears and locked themselves in the laboratories under similar circumstances... My own route, as you have gathered, was to go mad. (as cited in Anthony, 1991)

Pawley's account is likely extreme, but the stress associated with crits persists for many students today (Dannels \& Martin, 2008; AIAS Student Culture Task Force, 2002). Additional factors including chronic stressors, rumination over past events, and anticipation of upcoming events can also lead to poor sleep habits and poor sleep quality (e.g., Harvey, 2000; Scullin, Krueger, Ballard, Pruett, \& Bliwise, 2018; Söderström, Ekstedt, Åkerstedt, Nilsson, \& Axelsson, 2004).

\section{Sleep Education Programs and Other Interventions}

The literature on the cognitive, physical, and emotional effects of sleep deprivation, in conjunction with the literature on poor sleep habits in college students (including students in studio courses), suggests the need for sleep interventions. Some interior design programs have eliminated $24 / 7$ access to facilities, but currently, there are no available data on the effectiveness of this strategy for improving sleep. Another approach taken by high schools and universities is to educate students about the negative effects of poor sleep with the hope 
that doing so will change students' behaviors (e.g., Rigney et al., 2015; Gruber, 2016; Quan \& Ziporyn, 2017; Wing et al., 2015). Sleep education programs are successful at increasing students' awareness and knowledge of sleep; however, they do not typically change behaviors, such as total sleep duration (for review, see Blunden et al., 2012). For example, Rigney et al.'s (2015) randomized controlled trial investigated the effectiveness of a sleep education program in 296 students across 12 Australian middle schools. The intervention group received four classroom lessons and participated in a group project on sleep topics, which students presented at an evening showcase for parents. Following the education program, the intervention group's time in bed increased by 10 minutes, but these modest changes were not sustained in the 18-week follow-up. This "knowledge-behavior gap" has been observed in health domains as well (e.g., diet, exercise, drug use) (Gallasch \& Gradisar, 2007; Rimal, 2000; Sheeran \& Webb, 2016). As such, additional methods beyond education should be explored in order to motivate students to prioritize healthy sleep.

\section{Micro-incentives and Behavior Change}

Extra credit "micro-incentives" are small incentives that can be used to motivate behaviors (Dommeyer, Baum, Hanna, \& Chapman, 2004; Sundstrom, Hardin, \& Shaffer, 2016). For example, within the classroom environment, instructors frequently use micro-incentives to encourage higher response rates on course evaluations. Sundstrom et al. (2016) found that instructors who offered micro-incentives had more students fill out the course evaluation (84\%) compared to instructors who did not (51\%). Additional studies have demonstrated that small incentives can be effective in promoting healthy behaviors and practices (e.g., exercising, healthy eating) among college students (DeVahl, King, \& Williamson, 2005; McComb, Jones, Smith, Collins, \& Pope, 2016). Consider, for example, DeVahl et al.'s study in which 210 physical therapy students were offered extra credit for participating in a voluntary exercise program. The extra credit motivated students to pursue the opportunity and "experience the process of behavioral change," thereby potentially improving their future exercise behaviors and health outcomes (DeVahl et al., 2005, p. 297).

The current study applied the logic of using extra credit incentives for behavioral change to address two objectives. First, we hypothesized that an extra credit incentive would motivate undergraduate interior design majors (who lacked any sleep education) to sleep eight hours per night during the five nights leading up to their final project deadline. Second, we hypothesized that students who showed longer or more consistent sleep durations would show better final project performance.

\section{Methodology}

\section{Participants}

Participants were interior design majors in a CIDA-accredited interior design program at a private research university. Students enrolled in a Graphics II course during Spring 2017 were given the opportunity to opt in to the eight-hour challenge (procedure described below). Of the 28 students enrolled in Graphics II, 23 students opted in to the eight-hour challenge; however, one student was later removed from the analyses due to academic misconduct. Therefore, 22 (of 27) students attempted the eight-hour challenge. Twenty-six 
of the students also completed the Pittsburgh Sleep Quality Index (PSQI) (Buysse et al., 1989), which provides an estimate of global sleep quality over the previous month. According to their global scores on this questionnaire, $71 \%$ of the students would be classified as poor sleepers $(M=7.19, S D=2.80)$; only four students reported that they normally sleep 8 hours/night. These values are consistent with those reported by interior design students in our previous work (King et al., 2017) and are comparable to or worse than the levels of sleep quality found in large-scale studies of college students (e.g., Lund et al., 2010).

\section{Comparison Group}

Sleep in the eight-hour challenge students was compared to data from undergraduate interior design students who were previously monitored by actigraphy during their final project week at the same university. One sample included the 16 interior design students from King et al. (2017) who were completing a final project. To increase our comparison sample size proportionally to the eight-hour challenge opt-in group $(n=22)$, we monitored sleep in 6 additional interior design students before their final project deadline. The comparison group participants were enrolled regardless of their academic year, but academic year was not significantly related to total sleep time average $(r=-.23, p=.31)$ or total sleep time intraindividual variability $(r=.19, p=.40)$. The 22 non-incentivized comparison participants received monetary compensation for wearing the wristband sleep monitoring device for 7 nights, but we did not attempt to alter their normal sleeping patterns (cf. incentivized sleep in the eight-hour challenge group).

\section{Instructions for the Eight-Hour Sleep Challenge}

Seven days before the final project due date, students were issued the following extra credit opportunity via email:

"For 5 nights (Wednesday, April 26 to Monday, May 1) maintain an average of eight or more hours of sleep per night, with zero nights of sleep less than 7 hours, and we'll reward you with 12 POINTS EXTRA CREDIT on your final project. If you average between 7.0-7.99 hours per night, there will be no change to your final project grade. However, if you average less than 7 hours/night you will lose 5 points on your final project. Also, we want you to get good nighttime sleep, so naps don't count. This challenge is $100 \%$ elective; you do not have to participate. If you don't want to participate your grade will not be affected."

The reward for eight hours of sleep was included to motivate positive sleep behaviors (cf. Ajzen, 1985). In contrast, the penalty for short sleep was introduced to discourage allnighters and variable sleep patterns such as alternating between nights of long sleep and rebounding the next day, which are known to be detrimental to health and cognitive functioning (King et al., 2017; Phillips et al., 2017).

Participants wore actigraphy devices (Phillips Respironics - Actiwatch 2) to measure their sleep objectively. Actigraphy is a common tool in sleep research and uses a movement-based algorithm to estimate sleep/wake state objectively over several days or weeks (for review, see Ancoli-Israel et al., 2003). In healthy young adults, actigraphy has been shown to 
reliably estimate sleep duration relative to in-laboratory polysomnography measurement, which uses electroencephalography-derived brain waves to identify sleep (Ancoli-Israel et al., 2003; Kanady, Drummond, \& Mednick, 2011; Kripke et al., 2010; Van de Water, Holmes, \& Hurley, 2011). We employed standard, factory settings for data collection and analysis including 15-second epochs and medium threshold sensitivity (King et al., 2017).

Students wore the actigraphy device 24 hours per day for the entire assessment period. The device can detect nocturnal sleep as well as daytime sleep (i.e., napping), but only nocturnal sleep counted toward the eight-hour challenge (as communicated in the email to students). Sleep data collected by the actigraphy device includes time and date information, allowing researchers to differentiate napping and nocturnal sleep.

\section{Course Structure and Grading}

Graphics II is a first-year studio course on three-dimensional sketching and graphic representation. In Spring 2017, the two sections of this course were taught by different instructors, but they shared the same content, schedule, assignments, and grading rubrics. Both sections of Graphics II had their final project due on Monday, May 1, which was the day eight-hour challenge participants turned in their actigraphy device.

Throughout the course, students completed sketchbook assignments, field sketches, and completion exercises, but the majority of their grade was determined by four projects and a final project. Statistical analyses of the project grades indicated that the instructors' grading was reliable: internal consistency across the course projects showed acceptable reliability (Cronbach's alpha. = .77; Tavakol \& Dennick, 2011). Furthermore, students who scored well on the early projects were more likely to score well on the final project, $I(25)=.62, p<$. 001. The first four projects tested students' aptitude in each of the following areas: one-point perspectives, two-point perspectives, marker rendering, and watercolor rendering. The final project was comprehensive and worth $30 \%$ of the course grade. As such, the extra credit opportunity constituted $1.5 \%$ of the overall grade, which was somewhat higher than the traditional definition of an ECMI ( $<1 \%$ of grade; Dommeyer et al., 2004; Sundstrom et al., 2016). We used a larger incentive because we assumed that motivating first-year college students to change sleep behaviors before their final project would be much more challenging than the kinds of behavioral change for which ECMIs are typically used (e.g., turning in course evaluations).

To prevent bias in the evaluation of student work, the instructors graded the final projects using a standardized rubric that evaluated drawings (200 points, or 40 per drawing), final booklet (20 points), process work (50 points), and verbal presentation (30 points). The sleep data were analyzed by non-instructor research personnel and only made available to the instructors after all grading was completed. Therefore, when the instructors were grading projects they were masked to how well students had slept.

\section{Statistical Analyses}

We used planned $t$ tests and chi square analyses to compare sleep duration in participants in the eight-hour challenge group relative to participants in the non-incentivized comparison group. Wherever Levene's test was significant, we corrected the $t$ value and degrees of 
freedom for the unequal variances. To test the common student assumption that sleeping more before a major test or project impairs class performance (e.g., by taking time away from studying/working), we used $t$ tests to compare the class project scores of participants who opted in to the eight-hour challenge versus those who did not. Finally, we used Pearson $r$ correlations to examine whether total sleep duration and intraindividual variability in sleep duration (i.e., standard deviation of sleep durations) were associated with final project performance. We report Cohen's $d$ and phi as measures of effect size.

\section{Results}

Of the 264 possible nights of actigraphy data, there were 16 nights of missing data (i.e., $6 \%$ ). Missing data can occur due to technical failure (e.g., battery) or due to removing the wristband. If students were removing the wristband more in the eight-hour challenge group than in the non-incentivized group, then we would be concerned that students were doing so to inflate their average total sleep time. Importantly, however, missing data were nominally less common in the eight-hour challenge group $(M=4.6 \%)$ than in the non-incentivized comparison group $(M=7.1 \%), t(42)=0.56, p=.58, d=.17$. The following results are unchanged if excluding the three participants with missing data.

Each individual's mean sleep duration is illustrated in Figure 2. Figure 2 shows that students in the eight-hour sleep group $(M=7.99, S D=0.86)$ slept 98 minutes longer on average than students in the non-incentivized comparison group $(M=6.34, S D=1.15), t(42)=5.36, p<$. $001, d=1.65$. Their total sleep time was also a substantial improvement relative to how much these eight-hour challenge students self-reported that they normally sleep (i.e., on the PSQI; $M=6.69, S D=1.11), t(20)=4.60, p<.001, d=1.43$. When incentivized by the eight-hour challenge, students classified on the PSQI as poor quality sleepers were able to sleep $(M=8.18, S D=0.74)$ nominally better than students classified as good quality sleepers $(M=7.78, S D=0.91 ; t(19)=1.05, p=.31)$ Furthermore, students' intra-individual variability in sleep became more consistent: The standard deviation of sleep durations was much lower in the eight-hour sleep group $(M=0.95, S D=0.52)$ than in the non-incentivized comparison group $(M=1.80, S D=0.72), t(42)=4.48, p<.001, d=1.38$.

As illustrated in the infographic (figure 1), the eight-hour sleep challenge also improved the number of students who met optimal (eight hours) and minimal (seven hours) national sleep recommendations (e.g., Paruthi et al., 2016; Watson et al., 2015). There were six times the number of participants in the eight-hour sleep challenge (59\%) relative to the nonincentivized comparison group (9\%) that averaged eight hours per night, $\chi^{2}(1)=12.24, p<$. $001, \Phi=.53$. The results were even more impressive when using the minimum recommendation of seven hours per night (Watson et al., 2017). Only 14\% of students in the non-incentivized sleep comparison group averaged seven hours per night, but the eight-hour challenge increased this proportion to $86 \%$ of students, $\chi^{2}(1)=23.27, p<.001, \Phi=.73$. Figure 3 illustrates that, relative to participants' self-reported normal sleep times (PSQI), that $90.5 \%$ of students improved their sleep durations during the incentivized eight-hour challenge week; $38 \%$ of these students improved by at least 2 hours/night. 
A remaining question is whether taking the eight-hour challenge hurt final project performance; after all, students in the eight-hour challenge group reduced their time awake by 490 minutes on average across the week relative to students in the non-incentivized group (and an average of 413 minutes less than they reported on the PSQI to sleeping during the semester). In terms of academic performance, the students who opted in to the eight-hour challenge $(n=22)$ differed minimally, if at all, from the few students who did not take the challenge $(n=5)$. When averaging the four project grades that preceded the eight-hour challenge, students who later opted in to the challenge had similar grades $(M=90.20 \%, S D$ $=3.59)$ as students who did not opt in $(M=88.20 \%, S D=4.42), t(25)=1.08, p=.29, d=$ 0.43 . Furthermore, on the final project, students who took the challenge $(M=90.16 \%, S D=$ 4.79) performed nearly identically to students who did not take the challenge ( $M=90.65 \%$, $S D=1.72), t(19.20)=0.39, p=.70, d=0.14$ (corrected for unequal variances). Of course, in the context of this challenge in which extra credit was awarded to the best sleepers, when including final scores with extra credit, the students who opted in to the challenge ( $M=$ $95.06 \%, S D=9.08$ ) ended up with significantly better grades than those who did not, $t(24.89)=2.12, p=.04, d=0.68$.

Though the eight-hour challenge students slept well as a whole, some students maintained healthier sleep patterns than others. For example, some participants obtained adequate mean total sleep times by reducing sleep and then rebounding on sleep, which may explain why mean total sleep time was weakly and nonsignificantly related to final project grades $(I(20)$ $=.10, p=.65$ ). By contrast, Figure 4 demonstrates that students who maintained consistent sleep patterns (i.e., low intra-individual variability in sleep) showed significantly better final project grades than students who were inconsistent, $I(20)=-.45, p=.03$. Even after statistically controlling for mean total sleep time, consistent sleep patterns (i.e., low intraindividual variability in sleep) still predicted substantial variance in final project performance, $r(19)=-.49, p=.02$.

\section{Discussion}

Despite ample evidence supporting the health and cognitive benefits of healthy sleep, college students are known to engage in erratic sleep habits. And, during the week of final projects and exams (which can account for 20-30\% or more of a course), student sleep habits are (nearly) universally poor (Astill, Verhoeven, Vizjelaar, \& Someren, 2013; Zunhammer, Eichhammer, \& Busch, 2014). Our results, however, suggest that first-year college students can be incentivized to sleep for eight or seven hours per night by using an extra credit incentive, even when they have not received any sleep-specific education. Whereas only $9 \%$ of participants in the non-incentivized comparison group adhered to national sleep recommendations, $59 \%$ of eight-hour challenge students averaged eight hours of sleep per night. These results are encouraging in light of the literature on the "knowledge-behavior gap," especially research indicating minimal positive outcomes of sleep education interventions (for review, see Blunden et al., 2012). If educators wish students to sleep better during finals week, they can incentivize them to do so.

The putative view amongst students is that restricting sleep allows them to work more on a project (or study longer for a final exam). This view is based on at least two questionable 
assumptions: 1) that there are no other activities during the day that could be reduced (e.g., social activities, including using social media), and 2) that the efficiency and quality of one's work following restricted sleep durations is akin to that following recommended sleep durations. First, training students to manage their time is possible (e.g., Häfner, Stock, Pinneker, \& Ströhle, 2014), and time management training decreases stress levels in college students (Häfner, Stock, \& Oberst, 2015). Second, sleep restriction has been empirically demonstrated to decrease work productivity, sustained attention, and creativity (Budnick \& Barber, 2015; King et al., 2017). In studies of high school students, sacrificing sleep to spend more time studying did not benefit (and, in some cases, hurt) academic performance (Gillen-O'Neel, Huynh, \& Fuligni, 2013). Critically, in the present study of college students, the concern that sleeping more would come at a cost to final project performance was not substantiated: students who took the eight-hour challenge performed virtually identically to those who did not, and students who maintained good, consistent sleep patterns performed the best. The lack of academic benefit to restricting sleep, coupled with the known negative impacts of sleep loss on susceptibility to illness, automobile accidents, substance abuse, and mood dysregulation (Bryant \& Gomez, 2015; Haack \& Mullington, 2005; Prather, JanickiDeverts, Hall, \& Cohen, 2015), suggest the importance of a culture change for college students and educators.

Given that many interior design students currently accept irregular and shortened sleep as a regular part of studio culture, improving these habits will require a coordinated effort by faculty and administrators. The eight-hour challenge is one potential catalyst for change, but faculty should consider follow-up measures to encourage sustained healthy sleeping. Consider, for example, the recommendations from the American Academy of Pediatrics, the American Academy of Sleep Medicine, and the Centers for Disease Control and Prevention that classes should not start before 8:30 a.m. (Adolescent Sleep Working Group, 2014; Wheaton, Chapman, \& Croft, 2016). Research demonstrates that students perform better and retain more information with later start times (e.g., Carrell, Maghakian, \& West, 2011; Wahlstrom et al., 2014). Many researchers believe that even 8:30 a.m. is too early. For example, a recent analysis that incorporated sleep duration recommendations, circadian rhythms, and individual differences in chronotype concluded that 11 a.m. is the best time for classes (Evans, Kelley, \& Kelley, 2017). Beyond considering class start times, interior design programs should integrate discussions of sleep health, time management, and overall wellness into the curricula. By doing so, these programs can create a culture that not only advances academic outcomes, but also encourages practices that support mental and physical well-being.

\section{Limitations}

Our findings provide proof-of-principle that many studio-based majors can improve their sleep habits even while completing their final project. Still, some limitations are worth consideration. First, this was a cross-sectional, not an experimental, study due to ethical considerations not to randomly assign students to have or not have an extra credit opportunity. It is highly doubtful that over half of students would sleep for over eight hours per night without the challenge, though, because no published study has suggested that even 
$10 \%$ of students normally engage in such optimal sleep durations during finals week (e.g., Astill et al., 2013).

Second, the current study was limited to first-year undergraduate students majoring in interior design at a single university. Further research could apply a similar methodology at universities with a more diverse composition, or at least, to students in upper-level studios. As projects and work increase in scale, scope, and complexity, it could be that students require a larger incentive to sleep eight hours per night. Conversely, with greater experience in time management, students in upper-level studios may be even more successful in maintaining healthy sleep habits.

Third, actigraphy devices are expensive (approximately $\$ 1,000$ USD each), which limits the number of devices that can be purchased (and, consequently, limits the sample size of the current study). We do not recommend using sleep diaries (i.e., self-reported sleep) for the eight-hour challenge, because sleep diaries would be vulnerable to academic dishonesty, and it is already known that adults consistently overestimate their sleep duration (due to underestimating the time it takes to fall asleep and the duration of nighttime awakenings; King et al., 2017). A possible alternative for larger-scale studies is to rely on popular, commercial accelerometer devices (e.g., Fitbit). Commercial devices are often ${ }^{1} / 10$ of the expense of actigraphy devices, but are less accurate than actigraphy at discriminating sleep/ wake states. For example, Meltzer, Hiruma, Avis, Montgomery-Downs, and Valentin (2015) found that the Fitbit Ultra overestimated sleep duration by 41 minutes when in Normal mode, and underestimated sleep duration by 105 minutes when in Sensitive mode. Nevertheless, if the primary purpose is to motivate students to sleep better, and if students believe commercial devices or smartphone apps to be at least somewhat accurate accuracy, then those devices might be considered for interventional purposes (Bianchi, 2015).

\section{Implications}

The ultimate goal of the eight-hour challenge is to encourage students to work efficiently during the day and thereby reduce their need to work on projects late at night. The results suggest that providing students with an extra credit incentive increases the probability (sometimes dramatically) that they will adhere to minimal and optimal sleep durations. These results also suggest that students can adhere to recommended sleep durations without sacrificing academic performance.

The current study offers several future areas for research on improving student sleep habits. First, researchers need to determine the amount of extra credit points (or other incentives) that is most effective for motivating healthy sleep habits. Second, additional studies should follow up with eight-hour challenge participants in subsequent semesters to measure the sustained impact of the eight-hour challenge on work and sleep habits. If experiencing a positive behavior change in sleep in one's first year of college subsequently improves one's sleep health behaviors in later semesters, then the eight-hour challenge may be an intervention worth adopting broadly. 


\section{Acknowledgments}

The authors wish to thank Jason MacGregor and Kathy Hurtts for their generous sharing of actigraphy devices. M.K.S. was partially supported by NIH AG053161.

\section{References}

Adolescent Sleep Working Group, Committee on Adolescence, Council on School Health. (2014). School start times for adolescents. Pediatrics, 134, 642-649. [PubMed: 25156998]

Ahrberg K, Dresler M, Niedermaier S, Steiger A, \& Genzel L (2012). The interaction between sleep quality and academic performance. Journal of Psychiatric Research, 46(12), 1618-1622. [PubMed: 23040161]

Ajzen I (1985). From intentions to actions: A theory of planned behavior In Action control (pp. 1139). Springer Berlin Heidelberg.

American Institute of Architecture Students. Studio Culture Task Force., (2002). The redesign of studio culture: a report of the AIAS Studio Culture Task Force. Washington, DC: American Institute of Architecture Students.

Ancoli-Israel S, Cole R, Alessi C, Chambers M, Moorcroft W, \& Pollak CP (2003). The role of actigraphy in the study of sleep and circadian rhythms. Sleep, 26(3), 342-392. [PubMed: 12749557]

Anthony KH (1991). Design juries on trial: The renaissance of the design studio. New York: Van Nostrand Reinhold.

Astill RG, Verhoeven D, Vijzelaar RL, \& Someren EJ (2013). Chronic stress undermines the compensatory sleep efficiency increase in response to sleep restriction in adolescents. Journal of Sleep Research, 22(4), 373-379. [PubMed: 23398048]

Bachman L, \& Bachman C (2006). Student perceptions of academic workload in architectural education. Journal of Architectural and Planning Research, 23, 271-304.

Bianchi MT (2015). Consumer sleep apps: When it comes to the big picture, it's all about the frame. Journal of Clinical Sleep Medicine, 11(7), 695-696. [PubMed: 26094923]

Blunden SL, Chapman J, \& Rigney GA (2012). Are sleep education programs successful? The case for improved and consistent research efforts. Sleep Medicine Reviews, 16(4), 355-370. [PubMed: 22104441]

Bryant NB, \& Gómez RL (2015). The teen sleep loss epidemic: What can be done? Translational Issues in Psychological Science, 1(1), 116-125.

Buboltz WC Jr., Brown F, \& Soper B (2001). Sleep habits and patterns of college students: A preliminary study. Journal of American college health, 50(3), 131-135. [PubMed: 11765249]

Budnick CJ, \& Barber LK (2015). Behind sleepy eyes: Implications of sleep loss for organizations and employees. Translational Issues in Psychological Science, 1(1), 89-96.

Buysse DJ, Reynolds CF III, Monk TH, Berman SR, Kupfer DJ (1989). The Pittsburgh sleep quality index: A new instrument for psychiatric practice and research. Psychiatry Research, 28(2), 193213. [PubMed: 2748771]

Carrell SE, Maghakian T, \& West JE (2011). A's from Zzzz's? The causal effect of school start time on the academic achievement of adolescents. American Economic Journal: Economic Policy, 3(3), 62-81.

Carskadon MA, \& Dement WC (2017). Normal human sleep: An overview. Principles and practice of sleep medicine, 13-23.

Dannels DP, \& Martin KN (2008). Critiquing critiques: A genre analysis of feedback across novice to expert design studios. Journal of Business and Technical Communication, 22(2), 135-159.

Dempster FN, \& Farris R (1990). The spacing effect: Research and practice. Journal of Research and Development in Education, 23, 97-101.

DeVahl J, King R, \& Williamson JW (2005). Academic incentives for students can increase participation in and effectiveness of a physical activity program. Journal of American College Health, 53(6), 295-298. [PubMed: 15900994] 
Dommeyer CJ, Baum P, Hanna RW, \& Chapman KS (2004). Gathering faculty teaching evaluations by in-class and online surveys: their effects on response rates and evaluations. Assessment $\&$ Evaluation in Higher Education, 29(5), 611-623.

Evans MDR, Kelley P, \& Kelley J (2017). Identifying the best times for cognitive functioning using new methods: Matching university times to undergraduate chronotypes. Frontiers in Human Neuroscience, 11, 188. [PubMed: 28469566]

Gallasch J, \& Gradisar M (2007). Relationships between sleep knowledge, sleep practice and sleep quality. Sleep and Biological Rhythms, 5(1), 63-73.

Gillen-O’Neel C, Huynh VW, \& Fuligni AJ (2013). To study or to sleep? The academic costs of extra studying at the expense of sleep. Child Development, 84(1), 133-142. [PubMed: 22906052]

Gruber R (2016). School-based sleep education programs: A knowledge-to-action perspective regarding barriers, proposed solutions, and future directions. Sleep Medicine Reviews, 36, 13-28. [PubMed: 27818085]

Haack M, \& Mullington JM (2005). Sustained sleep restriction reduces emotional and physical wellbeing. Pain, 119(1-3), 56-64. [PubMed: 16297554]

Häfner A, Stock A, Pinneker L, \& Ströhle S (2014). Stress prevention through a time management training intervention: An experimental study. Educational Psychology, 34(3), 403-416.

Häfner A, Stock A, \& Oberst V (2015). Decreasing students' stress through time management training: An intervention study. European Journal of Psychology of Education, 30(1), 81-94.

Harvey AG (2000). Pre-sleep cognitive activity: A comparison of sleep-onset insomniacs and good sleepers. British Journal of Clinical Psychology, 39, 275-286. [PubMed: 11033749]

Hendricks JC, Sehgal A, \& Pack AI (2000). The need for a simple animal model to understand sleep. Progress in Neurobiology, 61(4), 339-351. [PubMed: 10727779]

Kanady JC, Drummond S, \& Mednick SC (2011). Actigraphic assessment of a polysomnographicrecorded nap: A validation study. Journal of Sleep Research, 20, 214-222. [PubMed: 20626612]

King E, Daunis M, Tami C, \& Scullin MK (2017). Sleep in studio based courses: Outcomes for creativity task performance. Journal of Interior Design, 42(4), 5-27.

Kripke DF, Hahn EK, Grizas AP, Wadiak KH, Loving RT, Poceta JS, .. \& Kline LE (2010). Wrist actigraphic scoring for sleep laboratory patients: Algorithm development. Journal of Sleep Research, 19(4), 612-619. [PubMed: 20408923]

Lesku JA, \& Rattenborg NC (2014). Avian sleep. Current Biology, 24(1), R12-R14. [PubMed: 24405667]

Lowe CJ, Safati A, \& Hall PA (2017). The neurocognitive consequences of sleep restriction: A metaanalytic review. Neuroscience \& Biobehavioral Reviews, 80, 586-604. [PubMed: 28757454]

Lund HG, Reider BD, Whiting AB, \& Prichard JR (2010). Sleep patterns and predictors of disturbed sleep in a large population of college students. Journal of Adolescent Health, 46(2), 124-132. [PubMed: 20113918]

McComb S, Jones C, Smith A, Collins W, \& Pope B (2016). Designing incentives to change behaviors: Examining college student intent toward healthy diets. Western Journal of Nursing Research, 38(9), 1094-1113. [PubMed: 27094555]

Meltzer LJ, Hiruma LS, Avis K, Montgomery-Downs H, \& Valentin J (2015). Comparison of a commercial accelerometer with polysomnography and actigraphy in children and adolescents. Sleep, 38(8), 1323-1330. [PubMed: 26118555]

Miyatsu T, Nguyen K, \& McDaniel MA (2018). Five popular study strategies: Their pitfalls and optimal implementations. Perspectives on Psychological Science, 13, 390-407. [PubMed: 29716455]

Nedeltcheva AV, Kessler L, Imperial J, \& Penev PD (2009). Exposure to recurrent sleep restriction in the setting of high caloric intake and physical inactivity results in increased insulin resistance and reduced glucose tolerance. The Journal of Clinical Endocrinology \& Metabolism, 94(9), 3242 3250. [PubMed: 19567526]

Paruthi S, Brooks LJ, D’Ambrosio C, Hall WA, Kotagal S, Lloyd RM, \& Rosen CL (2016). Recommended amount of sleep for pediatric populations: A consensus statement of the American Academy of Sleep Medicine. Journal of Clinical Sleep Medicine, 12(6), 785-786. [PubMed: 27250809] 
Phillips AJ, Clerx WM, O’Brien CS, Sano A, Barger LK, Picard RW, \& Czeisler CA (2017). Irregular sleep/wake patterns are associated with poorer academic performance and delayed circadian and sleep/wake timing. Scientific Reports, 7, 3216. [PubMed: 28607474]

Pilcher JJ, \& Walters AS (1997). How sleep deprivation affects psychological variables related to college students' cognitive performance. Journal of American College Health, 46(3), 121-126. [PubMed: 9394089]

Prather AA, Janicki-Deverts D, Hall MH, \& Cohen S (2015). Behaviorally assessed sleep and susceptibility to the common cold. Sleep, 38(9), 1353-1359. [PubMed: 26118561]

Quan SF, \& Ziporyn PS (2017). The impact of an online prematriculation sleep course (Sleep 101) on sleep knowledge and behaviors in college freshmen: A pilot study. Southwest Journal of Pulmonary and Sleep Medicine, 14, 159-163.

Rasch B, \& Born J (2013). About sleep's role in memory. Physiological Reviews, 93(2), 681-766. [PubMed: 23589831]

Rigney G, Blunden S, Maher C, Dollman J, Parvazian S, Matricciani L, \& Olds T (2015). Can a school-based sleep education programme improve sleep knowledge, hygiene and behaviours using a randomised controlled trial. Sleep Medicine, 16(6), 736-745. [PubMed: 25979180]

Rimal RN (2000). Closing the knowledge-behavior gap in health promotion: The mediating role of self-efficacy. Health Communication, 12(3), 219-237. [PubMed: 10938914]

Roane BM, \& Taylor DJ (2008). Adolescent insomnia as a risk factor for early adult depression and substance abuse. Sleep, 31(10), 1351-1356. [PubMed: 18853932]

Scullin MK (2017). Do older adults need sleep? A review of neuroimaging, sleep, and aging studies. Current Sleep Medicine Reports, 3(3), 204-214. [PubMed: 29226069]

Scullin MK, Krueger ML, Ballard HK, Pruett N, \& Bliwise DL (2018). The effects of bedtime writing on difficulty falling asleep: A polysomnographic study comparing to-do lists and completed activity lists. Journal of Experimental Psychology: General, 147(1), 139-146. [PubMed: 29058942]

Sheeran P, \& Webb TL (2016). The intention-behavior gap. Social and Personality Psychology Compass, 10(9), 503-518.

Smarr BL (2015). Digital sleep logs reveal potential impacts of modern temporal structure on class performance in different chronotypes. Journal of Biological Rhythms, 30(1), 61-67. [PubMed: 25564433]

Söderström M, Ekstedt M, Åkerstedt T, Nilsson J, \& Axelsson J (2004). Sleep and sleepiness in young individuals with high burnout scores. Sleep, 27(7), 1369-1377. [PubMed: 15586790]

Springett NR, \& Lekarz TK (1986). Faculty differences in psychological disturbance among undergraduates on arrival at university. Psychology and Psychotherapy: Theory, Research and Practice, 59(1), 69-73.

Sundstrom ED, Hardin EE, \& Shaffer MJ (2016). Extra credit micro-incentives and response rates for online course evaluations: Two quasi-experiments. Teaching of Psychology, 43(4), 276-284.

Tavakol M, \& Dennick R (2011). Making sense of Cronbach's alpha. International Journal of Medical Education, 2, 53-55. [PubMed: 28029643]

Taylor DJ, \& Bramoweth AD (2010). Patterns and consequences of inadequate sleep in college students: substance use and motor vehicle accidents. Journal of Adolescent Health, 46(6), 610 612. [PubMed: 20472221]

Thacher PV (2008). University students and the "All Nighter": Correlates and patterns of students' engagement in a single night of total sleep deprivation. Behavioral Sleep Medicine, 6(1), 16-31. [PubMed: 18412035]

Thomas M, Sing H, Belenky G, Holcomb H, Mayberg H, Dannals R, Redmond D (2000). Neural basis of alertness and cognitive performance impairments during sleepiness. I. Effects of $24 \mathrm{~h}$ of sleep deprivation on waking human regional brain activity. Journal of Sleep Research, 9(4), 335-352. [PubMed: 11123521]

Trockel MT, Barnes MD, \& Egget DL (2000). Health-related variables and academic performance among first-year college students: Implications for sleep and other behaviors. Journal of American College Health, 49(3), 125-131. [PubMed: 11125640] 
Trojanowski NF, \& Raizen DM (2016). Call it worm sleep. Trends in Neurosciences, 39(2), 54-62. [PubMed: 26747654]

Vandekerckhove M \& Cluydts R (2010). The emotional brain and sleep: An intimate relationship. Sleep Medicine Reviews, 14(4), 219-226. [PubMed: 20363166]

Van de Water A, Holmes A, \& Hurley DA (2011). Objective measurements of sleep for non-laboratory settings as alternatives to polysomnography-a systematic review. Journal of Sleep Research, 20, 183-200. [PubMed: 20374444]

Van Dongen HP, Maislin G, Mullington JM, \& Dinges DF (2003). The cumulative cost of additional wakefulness: Dose-response effects on neurobehavioral functions and sleep physiology from chronic sleep restriction and total sleep deprivation. Sleep, 26(2), 117-126. [PubMed: 12683469]

Wahlstrom K, Dretzke B, Gordon M, Peterson K, Edwards K, \& Gdula J (2014). Examining the Impact of Later School Start Times on the Health and Academic Performance of High School Students: A Multi-Site Study. Center for Applied Research and Educational Improvement. St. Paul, MN: University of Minnesota.

Walker M (2017). Why we sleep: Unlocking the power of sleep and dreams. Scribner.

Watson NF, Martin JL, Wise MS, Carden KA, Kirsch DB, Kristo DA, \& Rowley JA (2017). Delaying middle school and high school start times promotes student health and performance: An American Academy of Sleep Medicine position statement. Journal of Clinical Sleep Medicine, 13(4), 623625. [PubMed: 28416043]

Wheaton AG, Chapman DP, \& Croft JB (2016). School start times, sleep, behavioral, health, and academic outcomes: A review of the literature. Journal of School Health, 86(5), 363-381. [PubMed: 27040474]

Wing YK, Chan NY, Yu MWM, Lam SP, Zhang J, Li SX, \& Li AM (2015). A school-based sleep education program for adolescents: A cluster randomized trial. Pediatrics, 135(3), e635-e643. [PubMed: 25687152]

Wolfson AR, \& Carskadon MA (1998). Sleep schedules and daytime functioning in adolescents. Child Development, 69(4), 875-887. [PubMed: 9768476]

Wolfson AR, \& Carskadon MA (2003). Understanding adolescent's sleep patterns and school performance: A critical appraisal. Sleep Medicine Reviews, 7(6), 491-506. [PubMed: 15018092]

Zunhammer M, Eichhammer P, \& Busch V (2014). Sleep quality during exam stress: The role of alcohol, caffeine and nicotine. PloS One, 9(10), e109490. [PubMed: 25279939] 


\section{Healthy sleep is critical}

to factors that underlie professional and academic success:

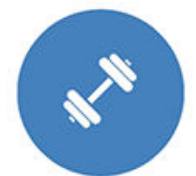

Physical health and wellness

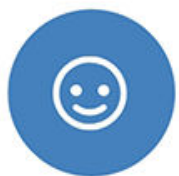

Emotional regulation

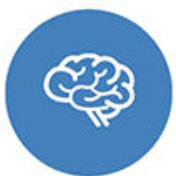

Cognitive

functioning

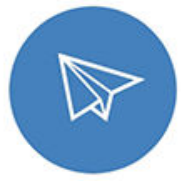

Creativity and problem solving

\section{Despite overwhelming
evidence supporting the need for quality sleep...

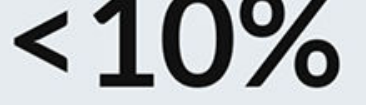 8 hours/night during finals.}

\section{Incentivizing healthy sleep before project deadlines:}

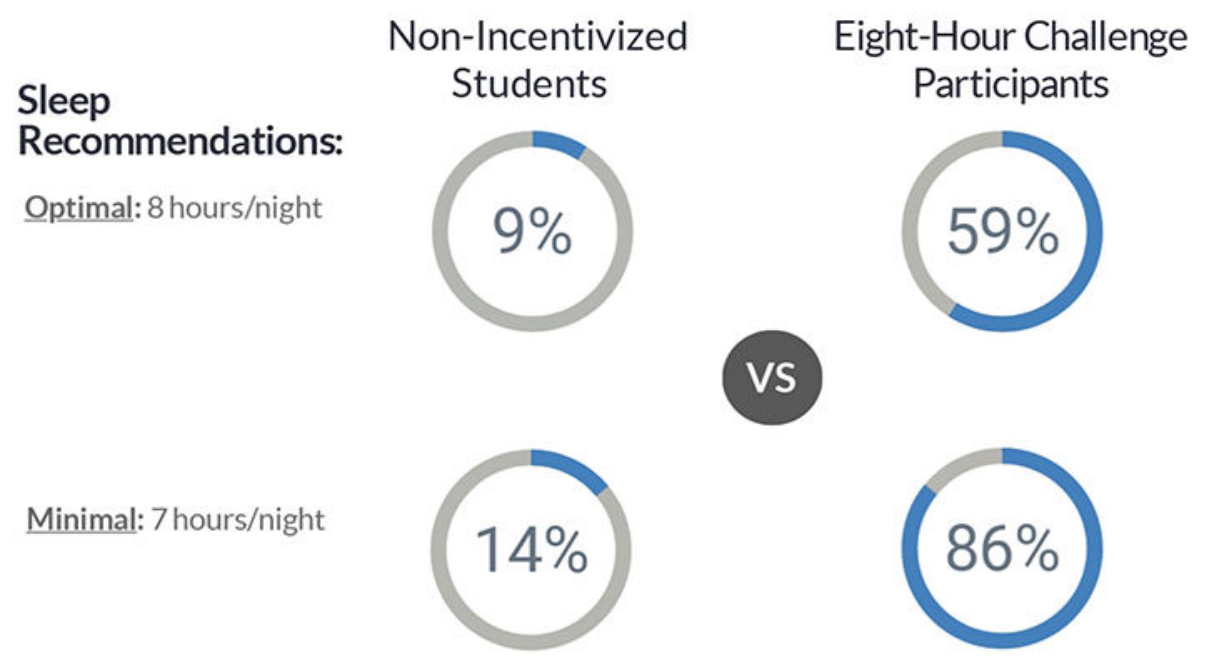

Figure 1. Infographic Summary of the Importance of Healthy Sleep and Comparison of EightHour Challenge Sleepers with Non-Incentivized Students.

More than six times the number of students in the eight-hour challenge group met optimal and minimal sleep recommendations compared to the non-incentivized comparison group. 


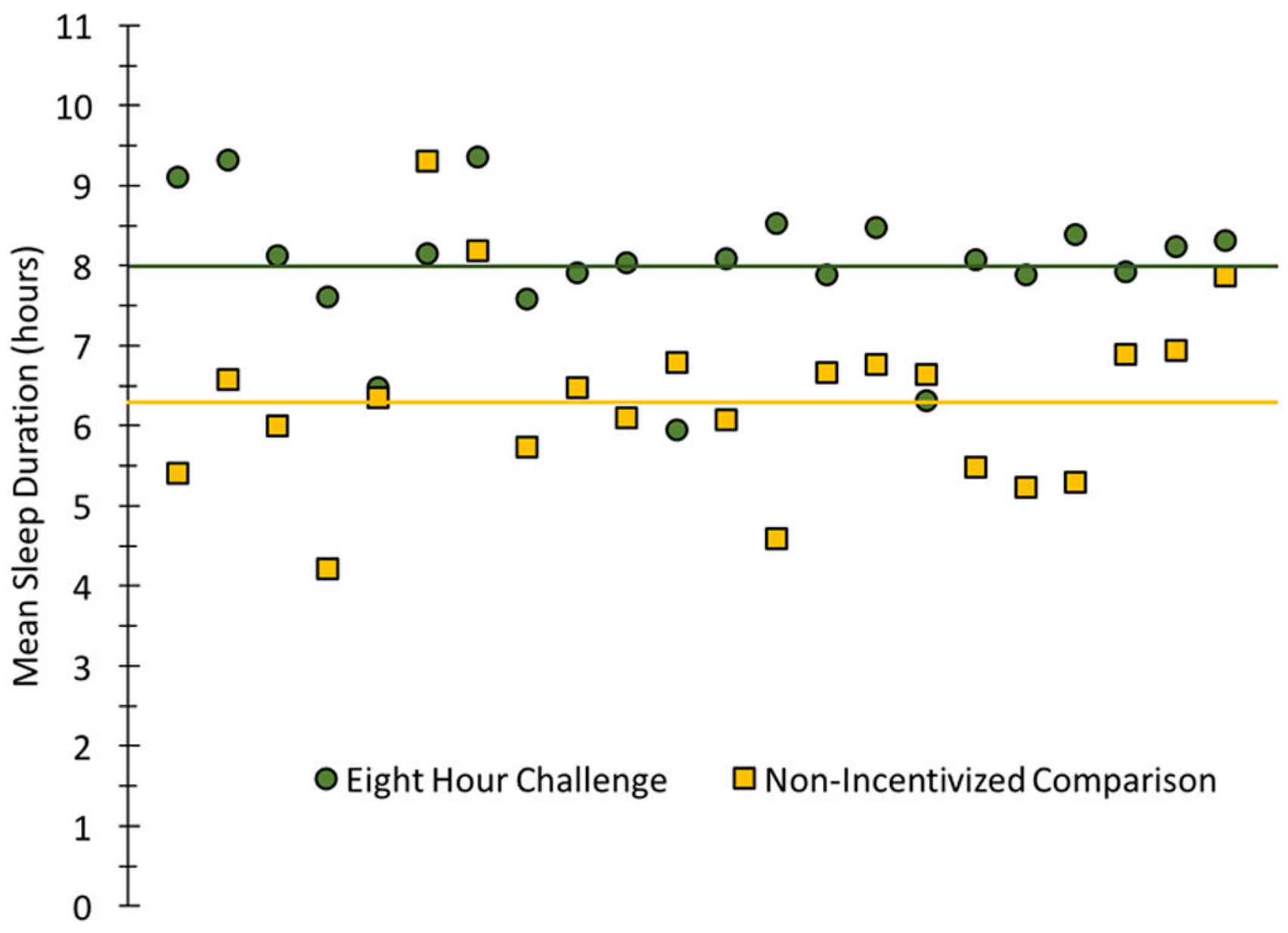

Figure 2. Mean Sleep Duration Preceding Submitting a Final/Term Project.

Each individual is represented by a green circle (Eight Hour Challenge group) or a gold square (Non-Incentivized Comparison group), with the group averages appearing as green and gold lines, respectively. 


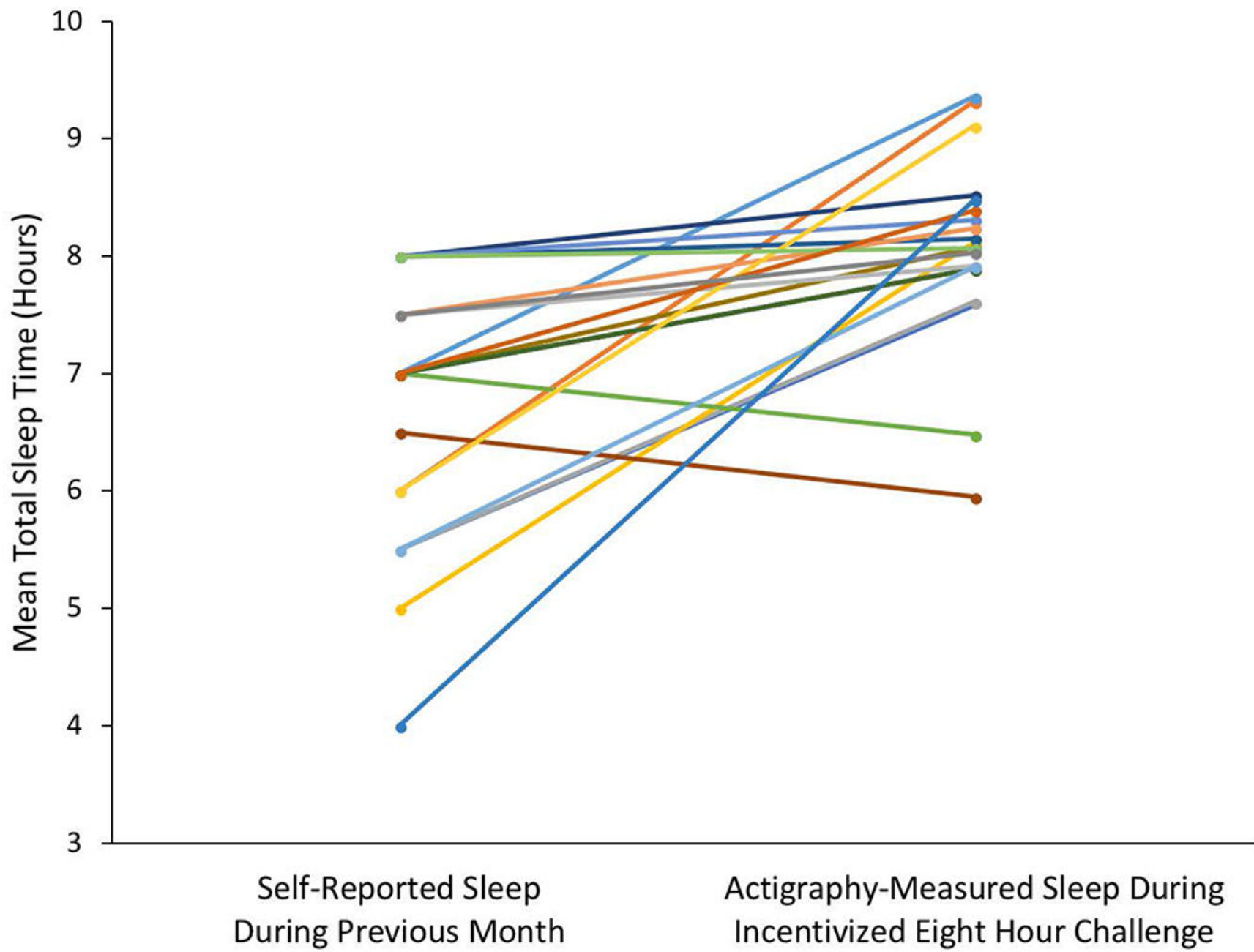

Figure 3. Changes in Sleep from Normal Weeks to Incentivized Week.

The majority of students were short and poor quality sleepers as assessed by the Pittsburgh Sleep Quality Index, but $90 \%$ of them improved their sleep when incentivized by extra credit (measured by actigraphy). 


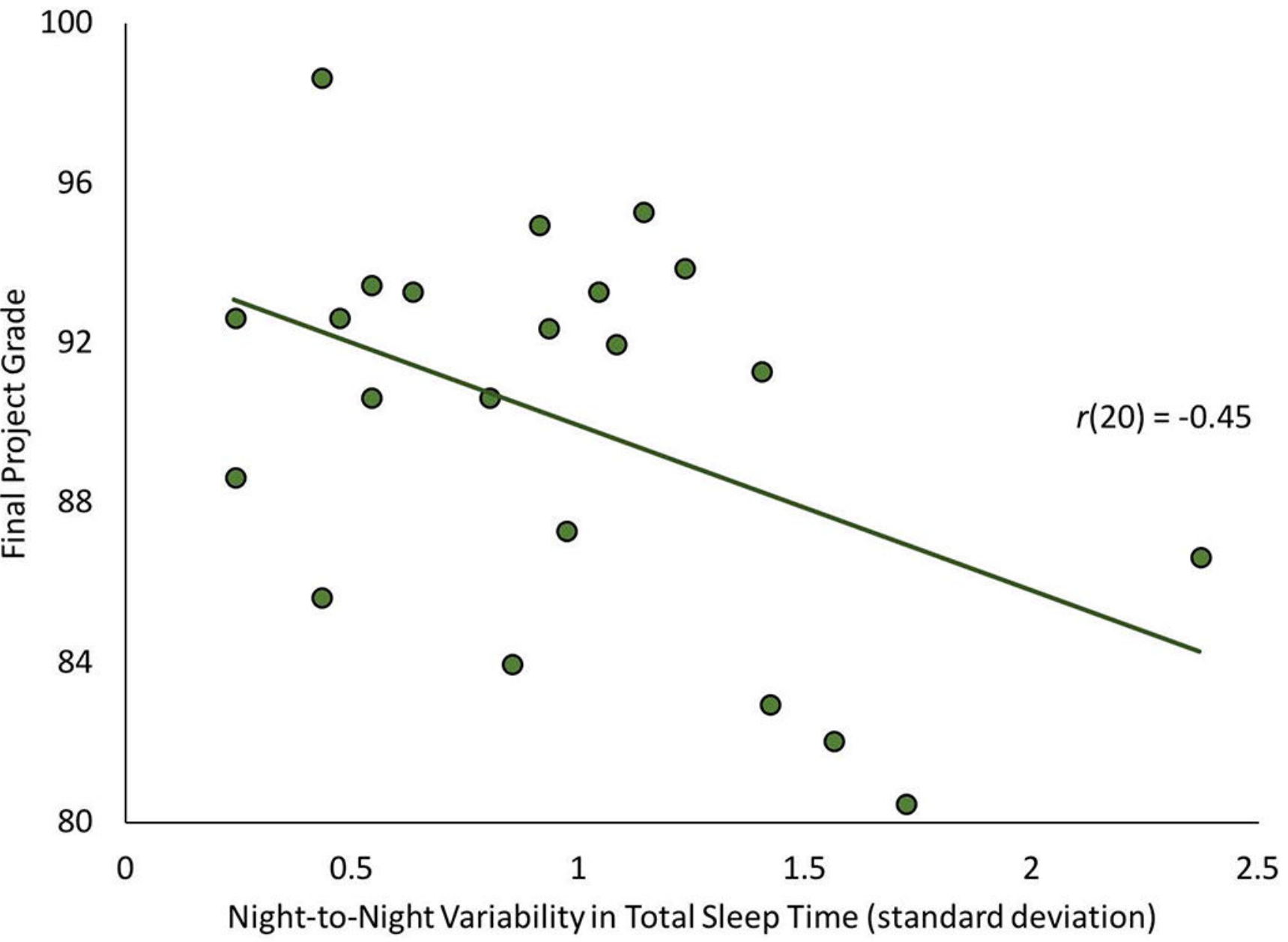

Figure 4. Greater Variability in Sleep Durations was Associated with Worse Final/Term Project Grade.

Scatterplot illustrates a negative association between standard deviations of sleep durations and final/term project grades in students who opted-in to the eight-hour challenge, an association that remained statistically significant even when controlling for mean total sleep time. 